Ciência Florestal, Santa Maria, v. 25, n. 2, p. 395-404, abr.-jun., 2015

ISSN 0103-9954

\title{
EXATIDÃO DE DENDRÔMETROS ÓPTICOS PARA DETERMINAÇÃO DO VOLUME DE ÁRVORES EM PÉ
}

\author{
ACCURACY OF OPTICAL DENDROMETERS FOR DETERMINING THE VOLUME \\ OF STANDING TREES
}

\author{
Marcos Felipe Nicoletti ${ }^{1}$ João Luis Ferreira Batista ${ }^{2}$ Samuel de Pádua Chaves Carvalho \\ Tito Nunes de Castro ${ }^{4}$ André Felipe Hess ${ }^{5}$
}

\begin{abstract}
RESUMO
O objetivo desse trabalho é verificar a exatidão dos dendrômetros ópticos Criterion 400 e RC3H, estudando a qualidade das medidas de cubagem da árvore em pé pelo uso desses dendrômetros. O trabalho foi desenvolvido na Estação Experimental de Itatinga - SP que pertence à Universidade de São Paulo (ESALQUSP). Amostraram-se no total 175 árvores em três parcelas de Eucalyptus grandis. O diâmetro do fuste das árvores em pé foi mensurado com os dois dendrômetros ópticos nas distâncias de 0,$1 ; 0,4 ; 0,7 ; 1,0 ; 1,3$; $2,0 \mathrm{~m}$ e a partir desse ponto, de metro em metro ao longo do tronco até os $8 \mathrm{~m}$ de altura. Após a cubagem em pé foram derrubadas as árvores e obtido o diâmetro nas diferentes posições com uma suta e trena nas mesmas posições em que se realizou a cubagem não destrutiva. De posse dos diâmetros foi calculado o volume por secção e por árvore individual pelo uso da fórmula de Smalian para posterior comparação dos métodos. Analisando as medidas do diâmetro do fuste e volume por árvore percebe-se que os dois dendrômetros forneceram medidas subestimadas de modo geral, porém, o Criterion foi o que resultou nas melhores estimativas. Para o diâmetro e volume por árvore, o Criterion demonstrou erros subestimados médios de aproximadamente $1 \mathrm{~cm}(10 \%)$, enquanto o RC3H resultou em erros superiores aos $5 \mathrm{~cm}(30 \%)$ em média. Portanto, quando se deseja maior confiabilidade e exatidão das variáveis observadas de forma não destrutiva, o Criterion apresentou melhores resultados.
\end{abstract}

Palavras-chave: dendrômetros ópticos; erros de medição; amostragem não destrutiva.

\section{ABSTRACT}

The aim of this paper is to verify the accuracy of the optical dendrometers Criterion 400 and $\mathrm{RC} 3 \mathrm{H}$ by studying the quality of measures of wood volume determination of standing trees through these dendrometers. The study was developed at the Experimental Station of Forest Sciences, in Itatinga, São Paulo state, which belongs to the University of São Paulo (ESALQ / USP). It was sampled a total of 175 trees in three plots of Eucalyptus grandis. The stem diameter of standing trees was measured by the two optical dendrometers at distances of $0.1,0.4,0.7,1.0,1.3,2.0$ meters from this point meter by meter along the stem up to 8 meters height. After measuring the standing trees they were felled and the diameter was obtained in different positions with a caliper and a tape in the same positions that the non-destructive measures were taken. With the diameters the volume was calculated by section and by individual trees by the Smalian formula for the comparison of methods. Analyzing the measurements of stem diameter and the individual tree volume

1 Engenheiro Florestal, MSc, Professor do Departamento de Engenharia Florestal, Universidade do Estado de Santa Catarina, Caixa Postal 281, CEP 88520-000, Lages (SC), Brasil. marcos.nicoletti@udesc.br

2 Engenheiro Florestal, PhD., Professor do Departamento de Ciências Florestais, Universidade de São Paulo, CEP 13418-900, Piracicaba (SP), Brasil. batista.jlf@usp.br

3 Engenheiro Florestal, Dr., Professor do Departamento de Engenharia Florestal, Universidade Federal de Mato Grosso, CEP 78060-900, Cuiabá (MT), Brasil. sam.padua@gmail.com

4 Engenheiro Florestal, MSc., Doutorando em Ciências pela Universidade de São Paulo, Av. Pádua Dias, 11, CEP 13418-900, Piracicaba (SP), Brasil. tncastro@gmail.com

5 Engenheiro Florestal, Dr., Professor do Departamento de Engenharia Florestal, Universidade do Estado de Santa Catarina, Caixa Postal 281, CEP 88520-000, Lages (SC), Brasil. andre.hess@udesc.br

Recebido para publicação em 22/10/2011 e aceito em 12/08/2013

Ci. Fl., v. 25, n. 2, abr.-jun., 2015 
realizes that the two dendrometers provided measures generally underestimated. However, the Criterion provided the best estimates. For the diameter and individual tree volume the Criterion showed underestimated errors averaging approximately $1 \mathrm{~cm}(10 \%)$, while the $\mathrm{RC} 3 \mathrm{H}$ resulted in errors greater than $5 \mathrm{~cm}(30 \%)$ on average.Thus, when measuring observed variables in a non-destructive way with reliability and accuracy, the Criterion showed better results.

Keywords: optical dendrometers; measurement errors; non-destructive sampling.

\section{INTRODUÇÃO}

A mensuração florestal é um importante elemento no manejo florestal, uma vez que fornece informações quali-quantitativas da floresta, permitindo, assim, tomadas de decisões mais precisas, além de possibilitar o melhor planejamento das atividades que norteiam esta área. Dentre das possíveis variáveis coletadas, as duas de maior relevância na realização de inventários florestais são, a altura e o diâmetro a altura do peito (DAP), que são usadas para o cálculo da área basal e do volume de madeira existentes em uma floresta (FREITAS e WICHERT, 1998).

Para a confiabilidade de um inventário florestal é necessário que se conheçam as suas fontes de erro, para assim tentar eliminar, ou ao menos minimizar, o seu efeito sobre a precisão das medições. Em um inventário florestal, os erros podem ser sistemáticos, relacionados com as medições, ou amostrais referentes ao sistema de amostragem utilizado (COUTO e BASTOS, 1988).

Segundo Perez (1989), os erros ocorridos nas medições dos diâmetros e alturas de árvores são devidos a erros do operador, defeitos no instrumento e nas condições de observação. Os erros cometidos na medição do DAP possuem uma maior ênfase quando comparado aos cometidos na medição da altura (COUTO et al., 1989).

Outra informação coletada em atividades de inventário é o volume das árvores. A cubagem rigorosa é um método tradicionalmente utilizado para quantificar o volume das árvores individuais. Para sua realização por meio de métodos destrutivos, torna-se necessária a derrubada das mesmas. Cubar uma árvore consiste em medir diâmetros a várias posições pré-definidas ao longo do fuste da árvore.

Essa prática como método destrutivo torna as atividades de inventário mais demoradas e com custos mais elevados, sendo particularmente problemática em florestas tropicais onde existem árvores de grande porte. Entretanto, há métodos que possibilitam a utilização de dendrômetros que realizam a mensuração dos diâmetros ao longo do fuste sob forma não destrutiva, como o Relascópio de Bitterlich, Pentaprisma de Wheeler, Criterion, entre outros. Desta forma, o uso desses equipamentos sob forma não destrutiva são fontes de dados para desenvolver diversas relações empíricas entre as variáveis observadas (ARNEY e PAINE, 1972; AVERY e BURKHART, 1997; CANALEZ et al. 2006; CARR, 1993; LIU et al., 1993; FREITAS e WICHERT, 1998; PARKEY e MATNEY, 1998).

O uso de relações empíricas é comum nas diversas áreas da ciência. Uma destas é o uso de equações de volume para obter o volume das árvores sem abatê-las, sendo que esse procedimento não é uma solução, pois o desenvolvimento da relação empírica exige os dados fornecidos pela cubagem rigorosa. Quando a relação empírica é extrapolada de uma região, onde foi desenvolvida via amostragem destrutiva, para outra região, onde a amostragem destrutiva não é possível, surge a questão da representatividade da relação empírica, com os problemas inerentes à extrapolação (CASTRO et al., 2008).

Nos inventários florestais é cada vez mais comum a utilização de equipamentos eletrônicos nas atividades de mensuração florestal, tais como: suta eletrônica, coletores de dados e medidores de altura. Isto se deve ao fato de que esses equipamentos facilitam a coleta de dados, assim como a posterior manipulação dos mesmos (FREITAS e WICHERT, 1998).

Sabe-se que existem no mercado inúmeros aparelhos capazes de medirem os diâmetros ao longo do fuste de forma indireta para o cálculo do volume (DITUNO, 1993; PARKEY, 1997). Observando-se que pelo uso dessa metodologia não há necessidade de derrubar as árvores, fato este que é de suma importância em áreas de conservação, para o grande potencial de fixação de carbono na biomassa das árvores e o crescente interesse global por este assunto. Tornando necessário o desenvolvimento de métodos de estimativa do teor de biomassa para verificar e adequar um método preciso na 
estimativa volumétrica por meio da cubagem não destrutiva de árvores, facilitando o planejamento e a valorização dos povoamentos. Diante desta tendência, o objetivo deste trabalho foi verificar a exatidão dos dendrômetros ópticos Criterion 400 e $\mathrm{RC} 3 \mathrm{H}$, por meio da comparação com o método destrutivo, a cubagem rigorosa, e avaliá-lo quanto a sua utilização em função do erro absoluto e relativo existentes nas medidas do diâmetro do fuste fornecidas pelos aparelhos; do volume por seção de cada torete; do volume acumulado das seções e do volume total em nível de árvores individualmente e de parcelas amostrais.

\section{MATERIAL E MÉTODO}

\section{Área de estudo}

O estudo foi realizado na Estação Experimental de Ciências Florestais de Itatinga do Departamento deCiências Florestais daUniversidade de São Paulo (ESALQ/USP). Localizada na região centro sul do Estado de São Paulo, a 2310'S e $48^{\circ} 40^{\prime} \mathrm{W}$, com 857 metros de altitude média. O clima da região, segundo Köppen, é caracterizado como mesotérmico úmido (Cwa), com precipitação anual média de $1308 \mathrm{~mm}$. A temperatura média do mês mais frio $12,8^{\circ} \mathrm{C}$ e a média do mês mais quente de $19,4^{\circ} \mathrm{C}$. As medidas de campo foram realizadas em um experimento de Eucalyptus grandis Maiden com 13 anos de idade e espaçamento $3 \times 2 \mathrm{~m}$.

\section{Descrição dos dendrômetros}

Foram utilizados dois dendrômetros de medição a laser para mensurar os diâmetros em diferentes alturas sob forma não destrutiva, o Criterion 400 e o RC3H da Savcor.

\section{Coleta de dados}

As medições foram feitas em três unidades amostrais de $360 \mathrm{~m}^{2}$, sendo que cada parcela possuía cerca de 60 árvores. Desta forma, foi mensurado um total de 175 árvores no povoamento de eucalipto. $\mathrm{O}$ resumo dos dados por parcela encontra-se na tabela a seguir.

As medidas dos diâmetros a diferentes alturas foram tomadas a $0,1 \mathrm{~m} ; 0,4 \mathrm{~m} ; 0,7 \mathrm{~m} ; 1,0 \mathrm{~m}$; $1,3 \mathrm{~m} ; 2,0 \mathrm{~m}$ e em intervalos de $1,0 \mathrm{~m}$ até a altura de $8,0 \mathrm{~m}$, totalizando doze posições por indivíduo. Este procedimento foi feito com os dendrômetros Criterion 400 e $\mathrm{RC} 3 \mathrm{H}$, sob forma não destrutiva. Para medir os diferentes diâmetros com o RC3H utilizou-se a vara telescópica (vara graduada) para delimitar a altura desejada. Fez-se a cubagem não destrutiva até $8,0 \mathrm{~m}$, pois em levantamentos preliminares, percebeu-se que a partir desse ponto os diâmetros obtidos já possuíam um erro considerável em relação ao diâmetro verdadeiro.

Depois de mensurados, os diâmetros com os dois dendrômetros com os indivíduos em pé e numerados com placa metálica, derrubaram-se as árvores com motosserra.

A cubagem rigorosa foi realizada com uso de uma trena e suta. Foram marcadas as mesmas medidas ao longo do fuste quando cubadas ainda em pé e a altura total. Após os 8,0 m, seguiram-se intervalos de $1,0 \mathrm{~m}$ nas seções do tronco até que o diâmetro fosse menor que $5,0 \mathrm{~cm}$. Para os diâmetros obtidos com a suta, foram feitas duas medidas em cada diâmetro, uma $90^{\circ}$ em relação à outra e obtida

TABELA 1: Resumo dos dados de diâmetro altura do peito (DAP), altura total (h) e volume total médio (v) por árvore em cada uma das três parcelas do povoamento de Eucalyptus grandis. Sendo que mín. e máx. representam o valor mínimo e máximo da variável de interesse observada na respectiva parcela.

TABLE 1: Summary of data in diameter at breast height (DAP), total height (h) and average total volume (v) per tree in each of the three portions of Eucalyptus grandis plantation. Since min. and max. represent the minimum and maximum values of the variable of interest observed in the respective plot.

\begin{tabular}{cccccccccc}
\hline \multirow{2}{*}{ Parcela } & \multicolumn{3}{c}{$\mathrm{DAP}(\mathrm{cm})$} & & \multicolumn{3}{c}{$\mathrm{h}(\mathrm{m})$} & \multicolumn{3}{c}{$\mathrm{v}\left(\mathrm{m}^{3}\right)$} \\
\cline { 2 - 9 } & mín. & médio & máx. & mín. & médio & máx. & mín. & médio & máx. \\
\hline 1 & 10,0 & 15,9 & 20,8 & 15,7 & 25,3 & 28,4 & 0,0532 & 0,2464 & 0,4582 \\
2 & 10,4 & 15,3 & 20,4 & 19,4 & 24,9 & 28,6 & 0,0794 & 0,2261 & 0,4394 \\
3 & 10,9 & 15,2 & 19,0 & 19,0 & 24,8 & 28,5 & 0,0932 & 0,2186 & 0,3668 \\
\hline
\end{tabular}


a média.

Volume parcial por seção ao longo do fuste e total

O volume individual por seção e das árvores foi calculado pela fórmula de Smalian, conforme Machado e Figueiredo Filho (2006). Desta forma, obteve-se o volume pelos três métodos, Criterion $400, \mathrm{RC} 3 \mathrm{H}$ e o volume considerado verdadeiro através da cubagem rigorosa. Para obter o volume a partir dos $8,0 \mathrm{~m}$ pelo Criterion 400 e pelo $\mathrm{RC} 3 \mathrm{H}$ usou-se o fator de forma (fi) calculado a seguir:

$$
\mathrm{f}_{\mathrm{i}}=\frac{\text { v.ponta }}{\mathrm{i}}
$$

Em que: $i=$ é o índice que se refere às árvores; v.ponta $i=$ é a diferença entre o volume sólido total $\mathrm{da}$ árvore $\left(\mathrm{m}^{3}\right)$ e o volume sólido até $8,0 \mathrm{~m}$ da árvore $\left(\mathrm{m}^{3}\right) ; d_{8 i}=$ diâmetro a $8,0 \mathrm{~m}$ de altura da cubagem rigorosa da árvore $(\mathrm{cm}) ; h t_{i}=$ altura total da árvore (m).

Assim, os volumes a partir de $8,0 \mathrm{~m}$ até a altura total $\left(\mathrm{vp}_{\mathrm{i}}\right)$ para os métodos do Criterion $400 \mathrm{e}$ $\mathrm{RC} 3 \mathrm{H}$ foram obtidos:

$$
\mathrm{vp}_{\mathrm{i}}=\left(\frac{\pi * \mathrm{~d}_{8 \mathrm{i}}{ }^{2}}{40000}\right) *\left(h \mathrm{t}_{\mathrm{i}}-8\right) * \mathrm{f}_{\mathrm{i}}
$$

Em que: $i=$ é o índice que se refere às árvores; $d_{8 i}=$ diâmetro a $8,0 \mathrm{~m}$ de altura da cubagem rigorosa da árvore $(\mathrm{cm}) ; h t_{i}=$ altura total da árvore $(\mathrm{m}) ; f_{i}=$ fator de forma da árvore.

Logo, os volumes totais pelo Criterion 400 e $\mathrm{RC} 3 \mathrm{H}$ foram obtidos pela soma dos volumes das seções até $8,0 \mathrm{~m}$ de altura e pelo volume da ponta $\left(v p_{\mathrm{i}}\right)$.

\section{Cálculo dos erros individuais}

Com os dados obtidos pelos três métodos foram calculados os erros absolutos e relativos para os diâmetros e para o volume por seção, acumulado e total. $\mathrm{O}$ erro absoluto da i-ésima árvore $\left(\mathrm{e} . \mathrm{ab} \mathrm{b}_{\mathrm{i}}\right)$ foi obtido da seguinte forma:

$$
\text { e. } a b_{i}=o b s_{i}-\operatorname{med}_{i}
$$

Em que: $i=$ é o índice que se refere às árvores; $o b s_{i}$ $=$ valor do diâmetro $(\mathrm{cm})$ ou volume observado $\left(\mathrm{m}^{3}\right)$ da árvore obtido pela cubagem rigorosa; med $_{i}=$ valor do diâmetro $(\mathrm{cm})$ ou volume medido $\left(\mathrm{m}^{3}\right)$ da árvore pelos métodos do Criterion 400 ou $\mathrm{RC} 3 \mathrm{H}$.

Logo, os erros relativos (e.re ${ }_{i}$ ) foram obtidos como descreve a fórmula abaixo.

$$
\text { e. } \mathrm{re}_{\mathrm{i}}=\left(\frac{\mathrm{e} \cdot \mathrm{ab} \mathrm{b}_{\mathrm{i}}}{\mathrm{obs}_{\mathrm{i}}}\right) * 100
$$

Em que: $i=$ é o índice que se refere às árvores; $e . a b_{i}$ $=$ erro absoluto do diâmetro $(\mathrm{cm})$ ou volume $\left(\mathrm{m}^{3}\right)$ da árvore; $o b s_{i}=$ valor do diâmetro $(\mathrm{cm})$ ou volume observado $\left(\mathrm{m}^{3}\right)$ da árvore obtido pela cubagem rigorosa.

O erro individual médio de cada árvore fornecido pelos diferentes dendrômetros foi comparado com as medidas fornecidas da cubagem rigorosa convencional. Sendo assim, os erros negativos indicam superestimativas e os erros positivos indicam subestimativas em relação ao volume verdadeiro.

\section{Erro no volume da parcela}

Para análise dos erros no cálculo do volume das parcelas utilizou-se o método Bootstrap. Este método foi introduzido por Efronem 1979 e implementado em Efron e Tibshirani (1993), no qual se faz o uso do método em diversas situações, envolvendo diferentes estimadores com respectivas variabilidades calculadas pelo método através de simulações computacionais. Leite (2007) comenta que a ideia básica do Bootstrap é reamostrar o conjunto disponível de dados para estimar os parâmetros por meio dos dados replicados, podendose assim obter a variabilidade dos mesmos sem pressupor nenhuma distribuição de probabilidade.

Portanto, neste trabalho tinha-se o volume gerado pelos três métodos diferentes, a cubagem rigorosa convencional, a cubagem com os diâmetros medidos com o Criterion e com os diâmetros medidos com o RC3H da Masser. Então, com o método Bootstrap foi reamostrado o conjunto de dados, do volume das três parcelas de cada método, para analisar o comportamento dos erros gerados em nível de unidade amostral pelo volume por hectare. Assim, foram geradas 1000 parcelas de 60 indivíduos para cada método, sem pressupor a distribuição de probabilidade qualquer. Assim, obtiveram-se valores médios do volume por hectare. 


\section{Análise dos dados}

Foi realizada a comparação entre os diferentes dendrômetros para determinação do volume de madeira das árvores. As comparações foram realizadas tanto no nível das árvores individuais quanto no nível das unidades amostrais. Os valores das diferenças entre as medidas geradas pelos dendrômetros ópticos e os valores obtidos pelo método destrutivo ou de medição direta do tronco foram obtidas por meio de estatísticas descritivas (média, desvio padrão) e de análises gráficas visando caracterizar a distribuição das diferenças. As análises foram realizadas por meio do software R (R DEVELOPMENT CORE TEAM, 2011).

\section{RESULTADOS E DISCUSSÃO}

Análise dos erros do diâmetro ao longo do tronco

A distribuição do erro absoluto das medidas dos diâmetros para os dois dendrômetros apresentou comportamentos distintos (Figura 1). As medidas provenientes do Criterion tiveram um comportamento quase que constante ao longo do fuste das árvores. Já aquelas resultantes do $\mathrm{RC} 3 \mathrm{H}$ apresentaram um erro crescente, conforme se aumenta a posição da medida dos diâmetros no fuste.

O Criterion teve em média um erro menor que $1 \mathrm{~cm}$ nas leituras, sendo que essas representam valores subestimados, ou seja, os diâmetros resultantes do dendrômetro sob forma não destrutiva forneceram medidas menores que as observadas na cubagem rigorosa. Parkey e Matney (1998) analisaram a utilização do Criterion 400 e outros dois dendrômetros ópticos para a obtenção de medidas de forma não destrutiva. Estes verificaram que os erros médios no diâmetro também foram subestimados em $0,13 \mathrm{~cm}$ com o Criterion 400 , em $0,86 \mathrm{~cm}$ com o Pentaprisma de Wheeler e para o Telerelascópio foi superestimado em $0,51 \mathrm{~cm}$. Para a altura encontraram erro médio de $15,2 \mathrm{~cm}$ para o Criterion 400 , de $48,8 \mathrm{~cm}$ para o Telerelascópio e de $51,8 \mathrm{~cm}$ com o Pentaprisma de Wheeler.

Logo, com o $\mathrm{RC} 3 \mathrm{H}$ observa-se um erro crescente superestimado para as posições mais próximas ao solo, até cerca de $2 \mathrm{~m}$ de altura. Já naquelas acima de $2 \mathrm{~m}$ do fuste, o erro continuou crescente conforme se aumentava a posição de medida no tronco. Essas, em contrapartida, tenderam a ser subestimadas em média até $5 \mathrm{~cm}$ no diâmetro aos $8 \mathrm{~m}$ de altura. Percebe-se que o erro absoluto do diâmetro com o $\mathrm{RC} 3 \mathrm{H}$ foi diretamente proporcional à medida que se aumenta a posição do fuste das árvores. Por isso, levantamentos com esse dendrômetro devem exigir maior cuidado na obtenção das suas variáveis e quanto ao nível de precisão exigidas nas suas medidas. Kalliovirta et al. (2005) avaliaram o funcionamento de outro instrumento capaz de realizar medidas indiretas do diâmetro, o Relascópio a laser, o qual é a combinação de um relascópio com um dendrômetro.
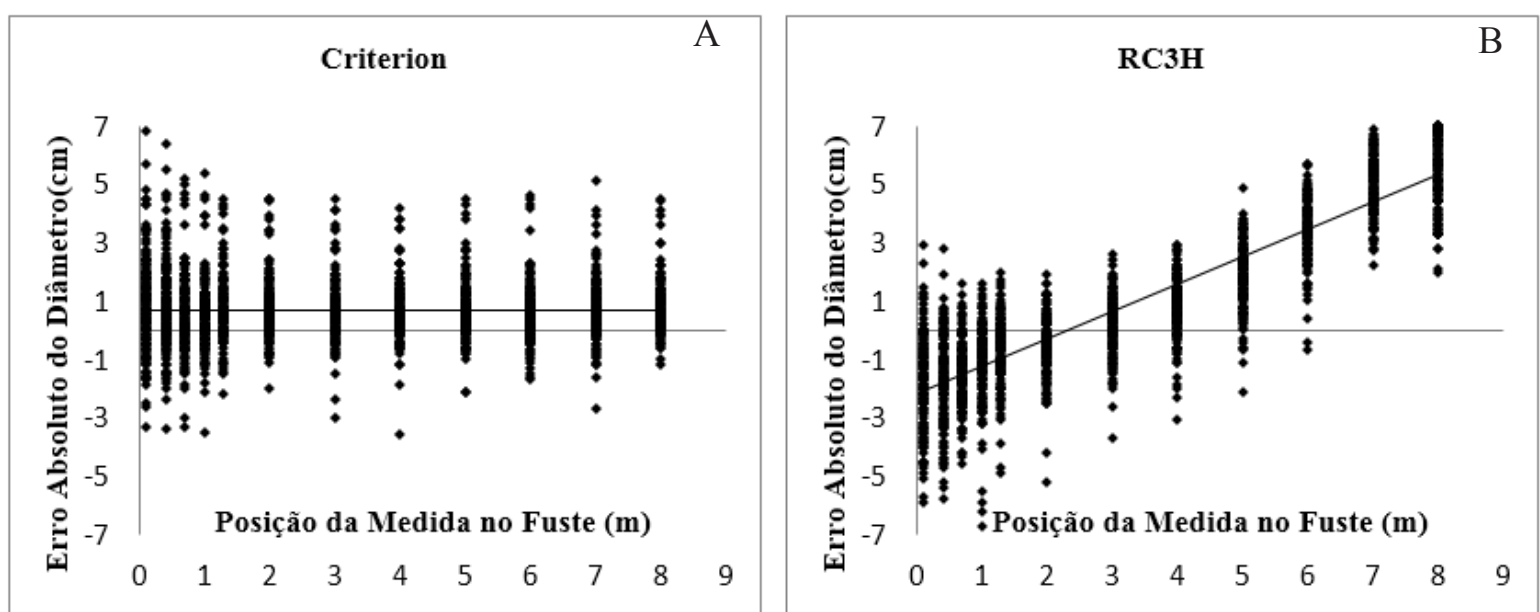

FIGURA 1: Erro absoluto do diâmetro $(\mathrm{cm})$ em relação à posição da medida do mesmo no fuste $(\mathrm{m})$ para o Criterion (A) e o RC3H (B) da Masser.

FIGURE 1: Absolute error of the diameter $(\mathrm{cm})$ compared to the same measure position at the stem $(\mathrm{m})$ to the Criterion (A) and $\mathrm{RC} 3 \mathrm{H}(\mathrm{B})$ of Masser. 
Comparando os resultados obtidos, concluíram que o Relascópio a laser demonstrou um erro padrão maior tanto para altura, como para o diâmetro em relação ao Criterion 400 e ao Barr \& Stroud.

Encontrou-se no erro relativo do diâmetro (Figura 2) neste trabalho, um comportamento semelhante ao erro absoluto. Nos diâmetros obtidos com a mensuração do Criterion observa-se que o erro relativo se manteve quase que constante ao longo do fuste. Esse erro foi inferior aos $10 \%$ nas diferentes posições ao longo do tronco, tendo como valor médio de 5\%. Williams et al. (1999) encontraram medidas ainda mais precisas do que a deste trabalho. Esses autores tiveram erros superestimados médios no diâmetro ao longo do fuste de $0,12 \mathrm{~cm}$, correspondendo a $0,40 \%$ e erro padrão de $1,43 \mathrm{~cm}$ para o Criterion. Diante desses valores, nota-se que as medições resultantes do Criterion parecem ser promissoras, devido a apresentarem baixos valores de erros médios. Fato que pode representar apenas um viés existente nas medições com o mesmo. Outro dendrômetro que demonstrou alta precisão nas medidas foi o Relascópio a laser, que segundo Kalliovirta et al. (2005) encontraram valores do DAP ligeiramente superestimadas em $0,13 \mathrm{~cm}$ $(0,7 \%)$ com erro padrão de $0,82 \mathrm{~cm}(5,9 \%)$, sendo essas, relativamente próximas às visualizadas neste trabalho. Em contrapartida, Freitas e Wichert (1998), testaram a utilização do Criterion 400 na obtenção de diâmetros e alturas de árvores e verificaram comportamento diverso aos apresentados. Esses autores encontraram erros de até $22 \mathrm{~cm}$ na medição do diâmetro com o Criterion, representando erros superestimados de aproximadamente $48 \%$.

Já para o RC3H, o erro relativo foi crescente ao longo do fuste, superestimado nas medidas até $2 \mathrm{~m}$ de altura e subestimado nos diâmetros acima dos $3 \mathrm{~m}$ de altura da planta. Nas medições acima de $6 \mathrm{~m}$, o erro relativo foi superior a $20 \%$ em média e continuou crescente. Diante desses, sabe-se que o $\mathrm{RC} 3 \mathrm{H}$ pode subestimar em até $40 \%$ aquelas tomadas em alturas mais elevadas. Couto et al. (1989), estudando os erros cometidos na medição do diâmetro e da altura de árvores, perceberam que um erro de $1 \mathrm{~cm}$ na estimativa do diâmetro correspondeu a um máximo de $19 \%$ no cálculo do volume.

Percebe-se também que, com o aumento das posições do fuste das árvores o erro tendeu a aumentar. Logo, nota-se que quanto mais alta for realizada a mensuração do diâmetro, maior será o erro proveniente da mesma para o $\mathrm{RC} 3 \mathrm{H}$. Fato este, que pode ter influência da distância que o observador se encontra da árvore e pelo aumento da dificuldade de visualização do diâmetro. Kalliovirta et al. (2005) e Williams et al. (1999) também concluíram que com o aumento da distância, observador-árvore, diminuiu a confiabilidade dos diâmetros obtidos com dendrômetros de tecnologia a laser.

Os principais fatores que exercem grande influência para acréscimo do erro nas medições não destrutivas segundo Kalliovirta et al. (2005), são a presença de líquens e a casca parcialmente solta no tronco, e em povoamentos muito densos e com alta ramificação. Já Williams et al. (1999) relatam
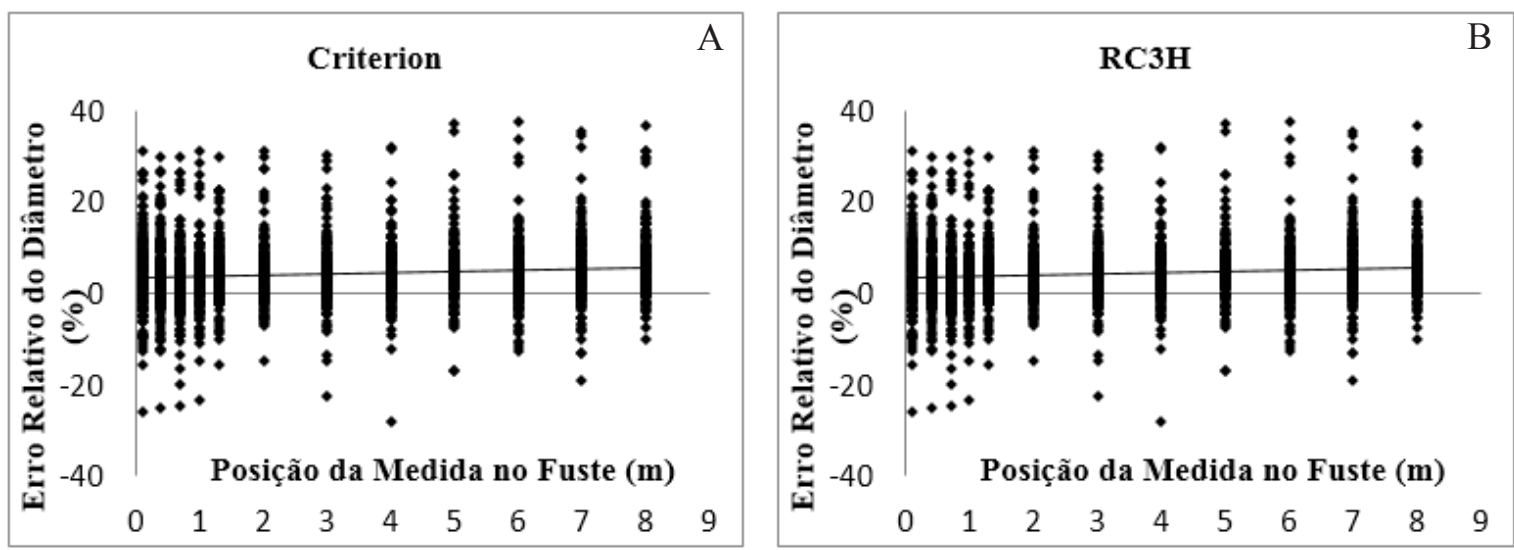

FIGURA 2: Erro relativo do diâmetro (\%) em relação à posição da medida do mesmo no fuste $(\mathrm{m})$ para $\mathrm{o}$ Criterion (A) e o RC3H (B) da Masser.

FIGURE 2: Relative error of the diameter (\%) compared to the same measure position at the stem (m) to the Criterion (A) and $\mathrm{RC} 3 \mathrm{H}(\mathrm{B})$ of Masser. 
que um dos maiores problemas na visualização do tronco das árvores com um relascópio a laser foi a paisagem que se encontra atrás desse indivíduo. Ou seja, quando o fundo for agrupado com fustes da mesma espécie, muitas vezes, dificulta a visualização das partes do tronco que se deseja mensurar. Como também as condições de luminosidade do povoamento também agravam esses problemas. Devem-se cuidar, então, os objetos indesejados que antecedem a árvore, pois o laser se interceptado por outro material fornecerá medidas deste e não daquele que realmente deseja-se mensurar.

Williams et al. (1999) verificaram também se o operador do instrumento exercia efeito significativo na acurácia das medidas com o Criterion 400 e com o Barr \& Stroud FP15. Esses perceberam que, independente do operador, as medidas do diâmetro e da altura pelo Barr \& Stroud apresentaram um erro sistemático superestimado. Entretanto, com o Criterion não houve uma tendência nas medidas, demonstrando assim, que o Barr \& Stroud exige uma calibração específica para que se evite tal erro.

Ferguson et al. (1984) comentaram que a calibração deve ser executada quando se deseja reduzir os erros sistemáticos causados pelo instrumento ou na modelagem dos dados. Clark et al. (2000) levantam que o viés do observador é muitas vezes fator-chave e pode afetar significantemente as medições, embora por mais comum que seja um efeito de instrumentação ou erro metodológico.

\section{Erros no Volume Acumulado}

Analisou-se o erro relativo do volume acumulado por torete (\%) em função da posição (m) em que os diâmetros foram obtidos para o Criterion e para o RC3H (Figura 3). Percebe-se que o comportamento do erro relativo foi semelhante aos encontrados para o diâmetro em função das posições do fuste. O Criterion novamente exibiu erros médios subestimados constantes inferiores aos $10 \%$ no volume acumulado por torete com o aumento das posições do tronco. $\mathrm{O} \mathrm{RC} 3 \mathrm{H}$ indicou mais uma vez o mesmo comportamento para o erro relativo no volume acumulado por torete (\%). Nas observações mais próximas ao solo, o erro médio superestimado resultou em média próximo dos $20 \%$. A partir dos $6 \mathrm{~m}$ de altura, o erro no volume acumulado por torete foi crescente e subestimado com o aumento da altura.

Sabe-se que o diâmetro é a variável independente mais importante nos modelos empíricos para a obtenção do volume. Quando observados os diâmetros com o Criterion, o erro registrado foi quase que constante ao longo do fuste das árvores, já os com o RC3H foram, na maioria das vezes, crescentes com o aumento das posições em que se mensurava o diâmetro. Portanto, percebe-se que a utilização do dendrômetro Criterion forneceu,
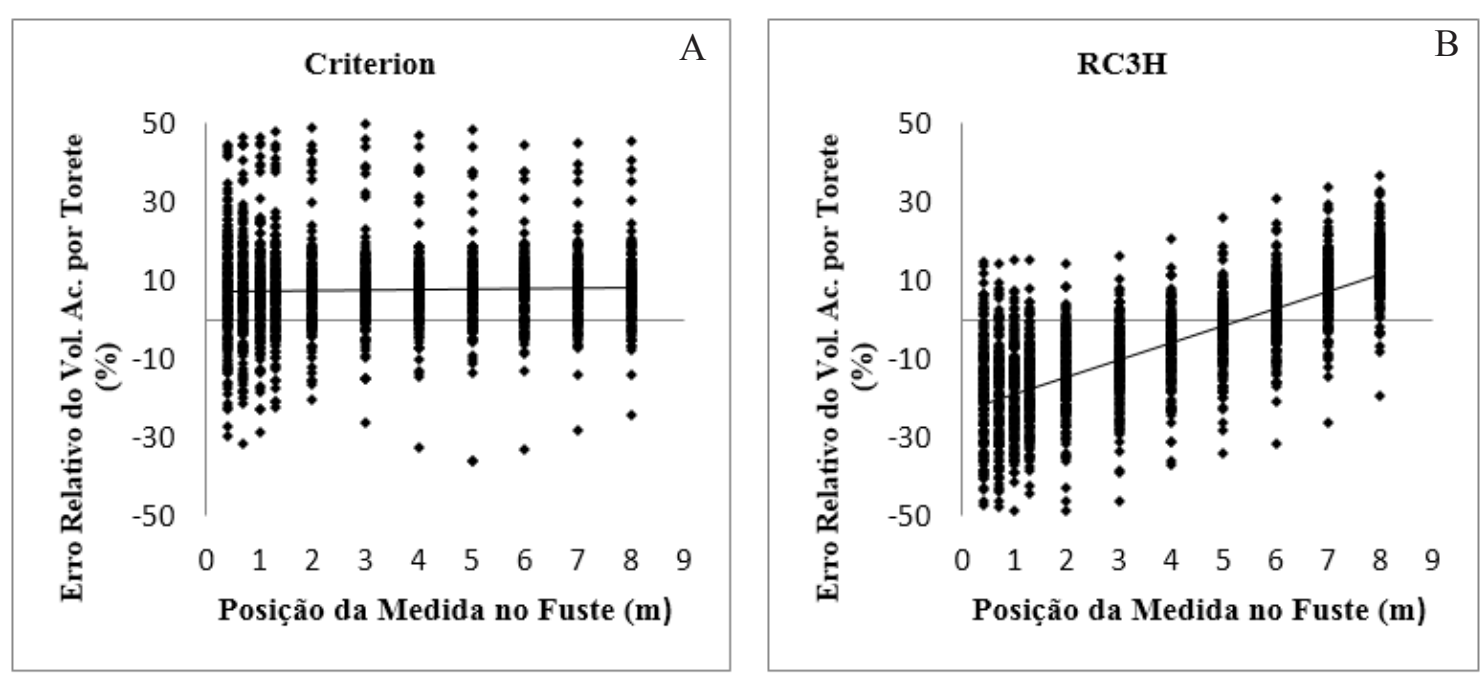

FIGURA 3: Erro relativo do volume acumulado por torete (\%) em função da posição (m) em que os diâmetros foram obtidos para o Criterion (A) e o RC3H (B).

FIGURE 3: Accumulated relative error of $\log$ volume $(\%)$ as a function of position $(\mathrm{m})$ in the diameter that was obtained for the Criterion (A) and $\mathrm{RC} 3 \mathrm{H}(\mathrm{B})$. 
de modo geral, estimativas mais precisas que o $\mathrm{RC} 3 \mathrm{H}$.

\section{Erro do Volume da Árvore}

O erro relativo no volume total (\%) calculado com o fator de forma apresentou um erro para o Criterion em torno dos 10\% (Figura 4). Em contrapartida, o erro relativo no volume total (\%) do $\mathrm{RC} 3 \mathrm{H}$ foi próximo dos $40 \%$ para o volume total das árvores. Desta forma, sabe-se que o dendrômetro que apresentou maior precisão no volume total das árvores foi o Criterion, devido ao erro médio de suas medidas.

Parkey e Matney (1998) também obtiveram as melhores estimativas com o Criterion 400, tanto para o diâmetro, altura e volume, quando compa-rado com o Pentaprisma de Wheeler e o Telerelascópio. Com o Criterion encontraram as menores variações volumétricas médias, cerca de 3,0\%, para o Pentaprisma de Wheeler de $8,9 \%$, ambas subestimadas, e por fim, valores superestimados para o Telerelascópio de 6,3\%.

\section{Erro no volume da parcela}

Por meio das simulações obtidas do método Bootstrap para a análise do comportamento do erro do volume total $\left(\mathrm{m}^{3} / \mathrm{ha}\right)$ observou-se grande diferença entre os métodos analisados (Figura 5).

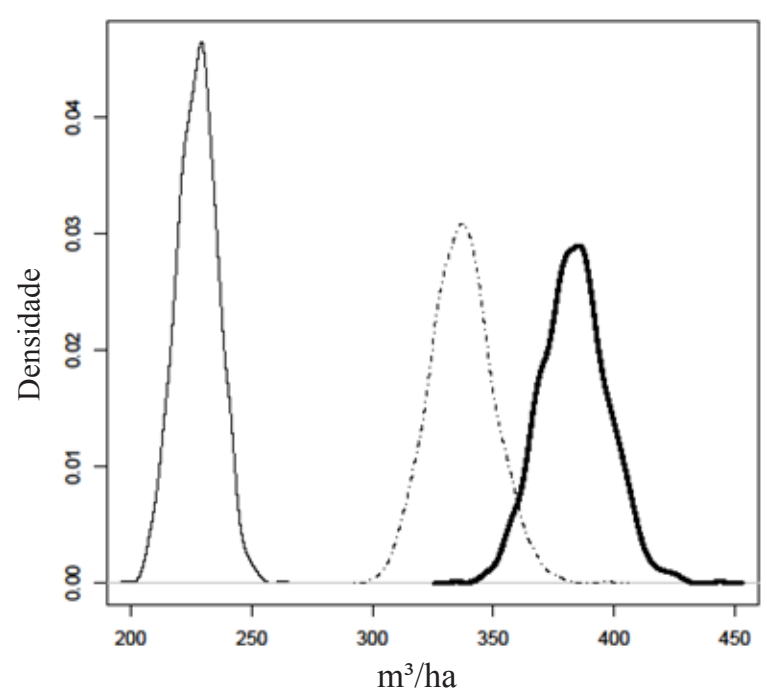

FIGURA 5: Curvas obtidas por meio das simulações do método Bootstrap representando o volume $\left(\mathrm{m}^{3} / \mathrm{ha}\right)$, fornecidos pela cubagem rigorosa (linha realçada), pelas medidas não destrutivas do dendrômetro Criterion (linha pontilhada) e do RC3H (linha normal).

FIGURE 5: Curves obtained by boot strap method simulations representing the volume $\left(\mathrm{m}^{3}\right)$, provided by destructive determination (bold line), the non-destructive measurements of dendrometer Criterion (dotted line) and $\mathrm{RC} 3 \mathrm{H}$ (normal line).
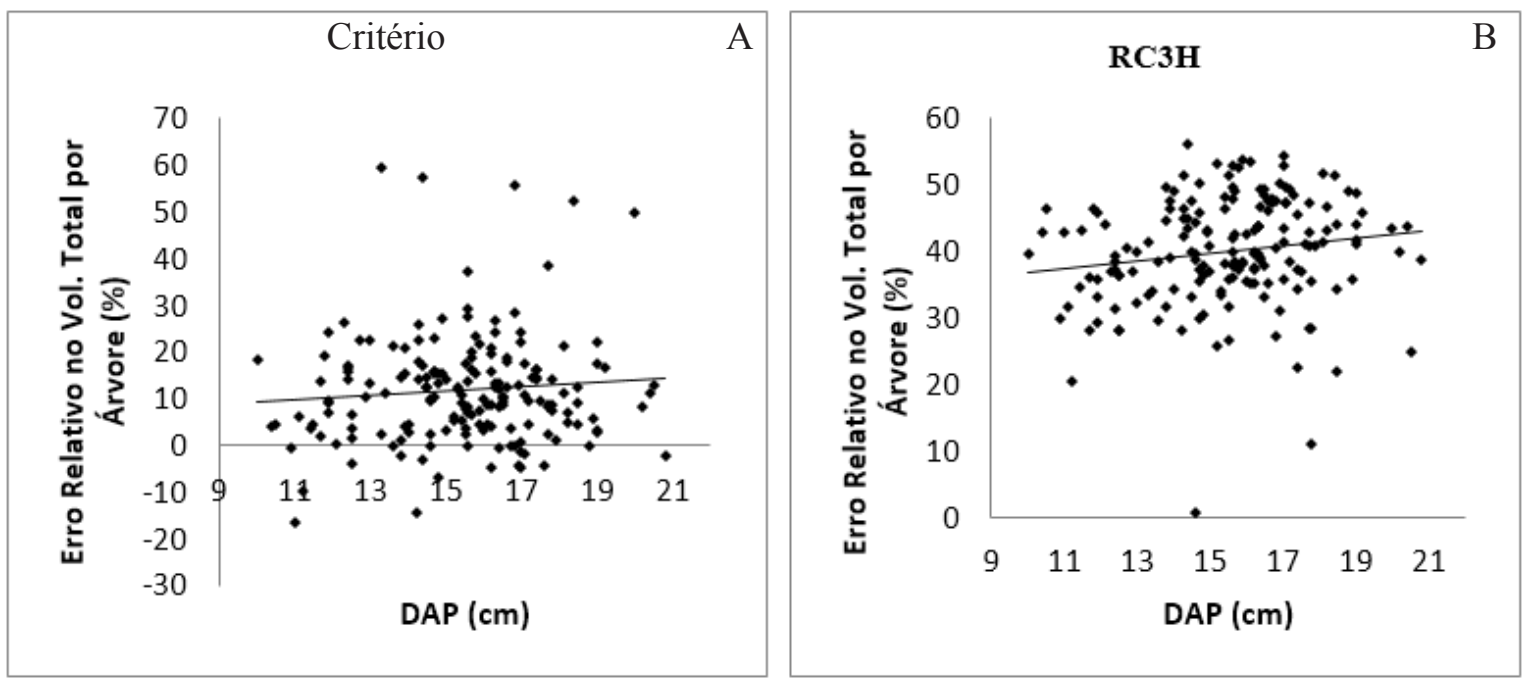

FIGURA 4: Erro relativo no volume total das árvores (\%) em função do DAP (cm) para os dendrômetros analisados.

FIGURE 4: Relative error in the total volume of trees $(\%)$ as a function of DBH $(\mathrm{cm})$ for the dendrometers analyzed. 
Pelo uso da cubagem rigorosa tradicional obteve-se por meio das simulações um intervalo do volume por hectare de aproximadamente 350 a $420 \mathrm{~m}^{3} / \mathrm{ha}$. Já as simulações com as medidas obtidas de forma não destrutiva pelos dois dendrômetros ópticos apresentaram volumes por hectare subestimados. $\mathrm{O}$ volume por hectare obtido com as variáveis do Criterion pelas simulações apresentou estimativas mais próximas das reais, da cubagem rigorosa, variando de aproximadamente 300 a $370 \mathrm{~m}^{3} / \mathrm{ha}$. Por fim, as simulações com os dados do $\mathrm{RC} 3 \mathrm{H}$ demonstraram uma subestimada acentuada em relação às da cubagem tradicional, variando em média de 200 a $250 \mathrm{~m}^{3} / \mathrm{ha}$. Deste modo, sabe-se que o Criterion representou melhor as estimativas de forma não destrutiva em relação ao volume por hectare verdadeiro deste povoamento. Kalliovirta et al. (2005) testando outro dendrômetro óptico, o Relascópio a Laser, para estimar o volume por hectare obtiveram uma superestimava de em média $2,2 \mathrm{~m}^{3} / \mathrm{ha}(1,4 \%)$ no volume e um erro padrão de $4,5 \mathrm{~m}^{3} / \mathrm{ha}(2,8 \%)$. Concluindo que esse erro foi dependente da área basal das plantas e com estimativas volumétricas precisas.

\section{CONCLUSÃO}

Diante dos resultados obtidos com os dois dendrômetros ópticos pode-se concluir que o Criterion é aquele que fornece as melhores estimativas, tanto para o diâmetro do fuste quanto para o volume da árvore e por hectare. Uma vantagem que o coloca à frente do $\mathrm{RC} 3 \mathrm{H}$ foi que o mesmo foi capaz de medir os diâmetros ao longo do fuste sem auxílio de vara graduada para delimitar a altura pré-definida. Outro fator foi que o Criterion pode medir diâmetros em alturas superiores ao $\mathrm{RC} 3 \mathrm{H}$. Enquanto esse se limitou às medições até 8 metros de altura na árvore, com erros já próximos aos $40 \%$ no diâmetro ao longo do fuste, $30 \%$ no volume por árvore e aproximadamente $40 \%$ para o volume por hectare. Logo, o Criterion apresentou em todos os casos medidas mais exatas que o $\mathrm{RC} 3 \mathrm{H}$, em torno de $10 \%$ para o diâmetro e para o volume da árvore, e aproximadamente $15 \%$ para o volume por hectare, quando comparadas à cubagem rigorosa. Portanto, sabendo que o erro amostral considerado em um inventário florestal geralmente deve ser menor que $10 \%$, o Criterion sob forma não destrutiva, forneceu boas estimativas com pouca diferença de erro.

\section{REFERÊNCIAS BIBLIOGRÁFICAS}

ARNEY, D. J.; PAINE, D. P. Tree and stand volumes using heightac cumulation and telescopic Spiegelrelaskop. Forest Science, Washington, v. 18, n. 2, p. 159-168, June 1972.

AVERY, T. E.; BURKHART, H. E. Forest measurements. $4^{\text {th }}$ ed. New York: McGrawHill, 1997. 408 p. (McGraw-Hill series in forest resources).

CASTRO, T. N.; MONTEIRO, D. R.; BATISTA, J. L. F. Avaliação do aparelho Criterion 400 na cubagem rigorosa de árvores em pé. In: SIMPÓSIO INTERNACIONAL DE INICIAÇÃO CIENTÍFICA DA UNIVERSIDADE DE SÃO PAULO, 16., 2008, Piracicaba. Anais..., São Paulo, 2008.

CANALEZ, G. G.; CORTE, A.P. D.; SANQUETTA, C. R. Dinâmica da estrutura da comunidade de lauráceas no período 1995-2004 em uma floresta de araucária no sul do estado do Paraná, Brasil. Ciência Florestal, Santa Maria, v. 16, n. 4, p. 357-367, 2006.

CARR, W. Use of laser technology for tree measurement - present use and future development. Paper presented at Modern methods of estimating tree and $\log$ volume and increment. In: CONFERENCE AND WORKSHOP (IUFRO S4.02).June 14-16, West Virginia University, Morgantown, WV, USA.8 p. 1993.

CLARK, N. A.; WYNNE, R. H.; SCHMOLDT, D. L. A review of past research on dendrometers. Forest Science. Washington, v. 46, n. 4, p. 570-576. 2000.

COUTO, H. T. Z.; BASTOS, N. L. M. "Erros na medição de altura em povoamentos de Eucalyptus em região planta". IPEF, Piracicaba, n. 39, p. 21-31, 1988.

COUTO, H. T. Z.; BATISTA, J. L. F.; RODRIGUES, L. C. E. "Mensuração e gerenciamento de pequenas florestas". Documentos florestais, Piracicaba, n. 5, p. 1-37, 1989.

DITUNO, E. Criterion model 400 use for strip cruising. Washington. Forest Service. 1993.

EFRON, B.; TIBSHIRANI, R. J. An introduction to the bootstrap. New York: Chapman \& Hall, $1993.436 \mathrm{p}$.

FERGUSON, I. S. et al. Calibrating dendrometersdor estimating tree volumes. Australian Forest Research. Canberra. v. 14, p. 253-263. 2000.

FREITAS, A. G.; WICHERT, M. C. P. Comparação entre instrumentos tradicionais de medição de diâmetro e altura com o Criterion 400. IPEF, 
Circular Técnica, Piracicaba, n. 188, p. 1-7, 1998. KALLIOVIRTA， J.; LAASASENAHO, J.; KANGAS, A. Evaluation of the Laser-relascope. Forest Ecologyand Management. Amsterdam, v. 20, p. 181-194, 2005.

LEITE, L. R. C. Uso do estimador de razão generalizado na condução do inventário florestal. Campo Grande/MS: 2007, Dissertação de Mestrado em Agronegócios. UFMS - UNB - UFG.

LIU, C. J.; HUANG, X.; EICHEMBERGER, J. K. Using laser technology for measuring trees. Paper presented at Modern Methods of estimating tree and log volume and increment conference and worshop (IUFRO S4.02). Junho 14-16, West Virginia University, Morgantown, WV, USA. 1993. 13p.

MACHADO, S. A.; FIGUEIREDO FILHO, A. Dendrometria. 2. ed. Guarapuava: Unicentro, 2006. 316 p.

PARKER, R. C. Nondestructive Sampling
Applications of the Tele-Relaskop in Forest Inventory. Southern Journal of Applied Forestry. Washington., v. 21, n. 2, p. 75-83, 1997.

PARKEY, R. C.; MARTNEY, T. G. Comparison of Optical Dendrometers for Prediction of Standing Tree Volume. Southern Journal of Applied Forestry. Oklahoma, v. 23, n. 2, p. 407-417, 1998.

R DEVELOPMENT CORE TEAM (2011). R:

A language and environment for statistical computing. $\mathrm{R}$ Foundation for Statistical Computing, Vienna, Austria. ISBN 3-900051-07-0, $<$ URL http://www.R-project.org >. Acesso em 27 de julho de 2011.

WILLIAMS, M. S. et al. Evaluation of the Barr \& Stroud FP15 and Criterion 400 Laser Dendrometers for Measuring Upper Stem Diameters and Heights. Forest Science. Washington, v. 45, n. 1, p. 53-61. 1999. 